\section{§8. Propagation of Magnetic Island Due to Self-induced Zonal Flow}

Uzawa, K. (JAEA), Ishizawa, A., Nakajima, N.

We have investigated the propagation of magnetic island caused by the drift-tearing mode by numerically solving a reduced set of two-fluid equations ${ }^{1)}$. It is found that the island propagates into the ion diamagnetic direction when the island growth is saturated, and the propagation velocity becomes small as the viscosity increases. We have found that the island phase velocity is approximately same as the $E \times B$ flow averaged inside the island at the saturation state. In Figure 1 the total $E \times B$ flow velocity and each Fourier component $\left\langle v_{E}^{(m)}\right\rangle=\left\langle\partial_{x} \phi_{m} e^{i 2 \pi m y}\right\rangle$ from $m=0$ to $m=2$ at saturation state are shown as a function of the viscosity. When the viscosity is around $5 \times 10^{-5}$, excitation of $m=1$ mode is comparable to that of the $m=0$ mode, and both modes affect the direction of the averaged $E \times B$ flow. Since the signs of zonal flow and other modes are opposite, the propagation directinon of the island is determined by the competition between them. The zonal flow component is slightly larger than other modes, and thus the total $E \times B$ flow directs toward the ion diamagnetic direction. It is observed that the averaged $E \times B$ flow velocity monotonically decreases as the viscosity reduces. The contribution of the zonal flow to the averaged $E \times B$ flow becomes dominant to those of other modes when the viscosity is smaller than $10^{-5}$. This indicates that the self-induced zonal flow plays an important role in determining the propagation direction when the viscosity is small.

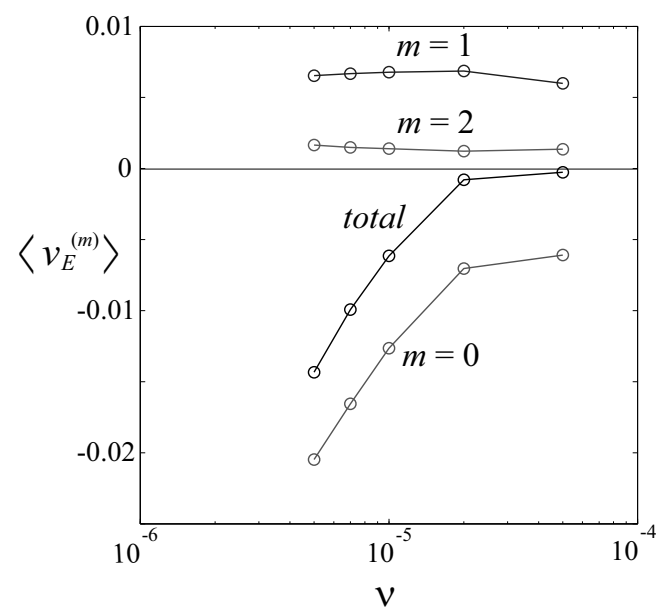

Fig. 1: The total $E \times B$ flow velocity $\left\langle v_{E}\right\rangle$ and each Fourier component $\left\langle v_{E}^{(m)}\right\rangle$ from $m=0$ to $m=2$.

We have examined the generation mechanism of the zonal flow and found that the contribution of each stress depends on the viscosity. Figure 2 plots contributions of each stress to zonal flow generation at saturation state as a function of the viscosity. The sum of contributions from the Reynolds stress $\left\langle v_{0}^{(R)}\right\rangle$, the Maxwell stress $\left\langle v_{0}^{(M)}\right\rangle$ and the viscous stress $\left\langle v_{0}^{(V)}\right\rangle$ is also plotted as a reference. Note that all contributions are averaged inside the island. The Reynolds stress is positive so that it forces the zonal flow into the equilibrium electron diamagnetic direction in all viscoisty regimes. On the contrary, the direction of the flow due to the Maxwell stress shows monotonically decreasing function with respect to the viscosity. When the viscosity is small, the Reynolds stress generates the zonal flow in the electron diamagnetic direction and the Maxwell stress almost cancels out the flow generation by the Reynolds stress. The small difference between them drives the flow in the electron diamagnetic direction. The flow velocity driven by the ion diamagnetic stress is negative so that it directs in ion diamagnetic direction. The direction of the flow due to the Maxwell stress is negative for small viscosity cases and is positive for large viscosity cases. The flow velocity driven by viscous stress is negative and is very small when the viscosity is small. The balance of these stresses depends on the viscosity. When the viscosity is small, the Reynolds stress and the Maxwell stress almost cancel out each other, and the small difference between them $\left\langle v_{0}^{(R)}\right\rangle+\left\langle v_{0}^{(M)}\right\rangle+\left\langle v_{0}^{(V)}\right\rangle$ is positive and drives the flow in the electron diamagnetic direction. The flow due to the ion diamagnetic stress is larger than this flow, as a consequence the zonal flow directs toward the ion diamagnetic direction when the viscosity is small. On the other hand, when the viscosity is large, the viscous stress is large, and it is counteracted by the Maxwell and the Reynolds stresses. The difference between them $\left\langle v_{0}^{(R)}\right\rangle+\left\langle v_{0}^{(M)}\right\rangle+\left\langle v_{0}^{(V)}\right\rangle$ drives the flow in the electron diamagnetic direction. The ion diamagnetic stress almost cancels out the flow but is slightly larger than the flow, and thus the zonal flow is very small and directs toward the ion diamagnetic direction when the viscosity is large.

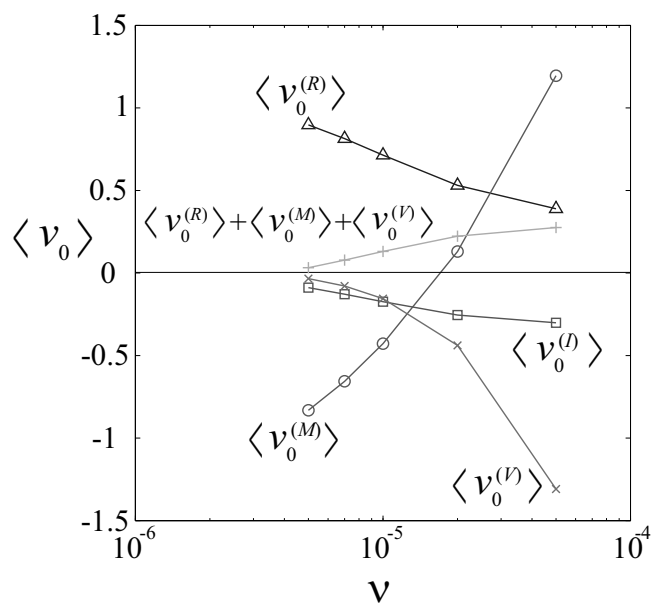

Fig. 2: Contributions of each stress to zonal flow generation in saturation regime as a function of the viscosity. Note that all contributions are averaged inside the island.

1) Uzawa, K. et al. : Phys. Plasmas 17 (2010) 042508 